\title{
The relationship between healthcare expenditure and disposable personal income in the US states: a fractional integration and cointegration analysis
}

\author{
Guglielmo Maria Caporale ${ }^{1}$ - Juncal Cunado ${ }^{2}$. \\ Luis A. Gil-Alana ${ }^{2}$ - Rangan Gupta ${ }^{3}$
}

\begin{abstract}
This study examines the relationship between healthcare expenditure and disposable income in the 50 US states over the period 1966-2009 using fractional integration and cointegration techniques. The degree of integration and nonlinearity of both series are found to vary considerably across states, while the fractional cointegration analysis suggests that a long-run relationship exists between them in only 11 out of the 50 US states. The estimated long-run income elasticity of healthcare expenditure suggests that health care is a luxury good in these states. By contrast, the short-run elasticity obtained from the regressions in first differences is in the range $(0,1)$ for most US states, which suggests that health care is a necessity good instead. The implications of these results for health policy are also discussed.
\end{abstract}

Keywords Healthcare expenditure · Income elasticity · US states · Fractional integration $\cdot$ Fractional cointegration

Mathematics Subject Classification C22 $\cdot$ C32 $\cdot$ H51 $\cdot$ I18

Luis Gil-Alana gratefully acknowledges financial support from the Ministerio de Economía y Competitividad (ECO2014-55236). Juncal Cuñado gratefully acknowledges financial support from the Ministerio de Economía y Competitividad (ECO2014-55496). Comments from the Editor and an anonymous referee are gratefully acknowledged.

$凶 \quad$ Juncal Cunado jcunado@unav.es

1 Brunel University London, London, UK

2 Department of Economics, University of Navarra, Pamplona, Spain

3 University of Pretoria, Pretoria, South Africa 


\section{Introduction}

According to the OECD Health Statistics (2014), in 2012 the USA spent $16.9 \%$ of its GDP on health care, which is far higher than the OECD average of 9.3\%, while on a per capita basis it spent more than double the OECD average. Furthermore, from 1960, US healthcare expenditure has grown five times faster than GDP (from $7.1 \%$ in the late sixties to $16.9 \%$ in 2012), and faster than in other OECD countries, and is projected to grow at an average rate of $5.7 \%$ until $2023,1.1 \%$ age points faster than the expected average annual growth rate of GDP. However, its level and growth rate have not been homogeneous across the US states, as pointed out in different papers analysing regional convergence in health spending (Wang 2009; Panopoulou and Patenlidis 2012, 2013). For instance, in 2009 per capita personal healthcare spending in Massachusetts $(\$ 9,278)$ was almost twice than in Utah $(\$ 5,031)$.

The existing literature (starting with Kleiman 1974 and Newhouse 1977) has suggested that disposable income, together with other demand and supply factors such as medical technological progress or demographic trends, is one of the key drivers of healthcare demand and therefore expenditure. However, the evidence on the existence of a long-run relationship between income and healthcare expenditure, as well as the income elasticity of healthcare expenditure and the relative importance of income as one of its drivers, is mixed (see, for example, Acemoglu et al. 2013; Wang 2009; Freeman 2012; Yavuz et al. 2013, among others). Whether health expenditure is a luxury (income elasticity above 1) or a necessity (income elasticity below 1) good has important policy implications: in the latter case, there is a strong argument for public health policies and more public involvement (Freeman 2012).

The contribution of this paper is threefold. First, we examine the long-memory properties of healthcare expenditure and disposable income in 50 US states, allowing for nonlinear deterministic trends in the form of Chebyshev polynomials. We take a fractional integration approach that has advantages relative to the standard unit root tests previously used, given the low power of the latter in the case of fractional and near unit root processes (see for example, Diebold and Rudebusch 1991; Hasslers and Wolters 1994; Lee and Schmidt 1996; and more recently, Ben Nasr et al. 2014). However, it is well known that the presence of structural breaks in the data can lead to spurious evidence of long memory (see for example, Cheung and Lai 1993; Diebold and Inoue 2001; Ben Nasr et al. 2014). Given the existing evidence suggesting the presence of structural breaks in both healthcare expenditure and personal income (Freeman 2012) and the small sample size in our study (forty-four annual observations, 1966-2009), we model them including nonlinear time trends in the form of Chebyshev polynomials; this approach is particularly appropriate at the annual frequency, for which the breaks are likely to be smooth rather than sharp and sudden, and does not require specifying a maximum number of breaks when testing for unit roots. Second, we analyse the long-run relationship between income and healthcare expenditure using both parametric (Gil-Alana 2003) and semiparametric (Robinson 1995a; Marinucci and Robinson 2001) methods to test for fractional cointegration. To our knowledge, this is the first study applying such methods for estimating the relationship between these two variables in the US states. Third, we obtain estimates of the income elasticity 
with the aim of establishing whether health care should be considered a luxury or a necessity good in each of the US states.

The remainder of the paper is structured as follows. Section 2 reviews the relevant literature. Section 3 describes the data and the empirical analysis. Section 4 summarises the main findings and discusses their policy implications.

\section{Literature review}

The relationship between healthcare expenditure (HCE) and disposable income has been extensively examined given its important policy implications. Estimates of income elasticities range from close to zero and below one (Di Matteo 2003; Baltagi and Moscone 2010; Freeman 2012) to higher than one (Ang 2010; Liu et al. 2010), depending on the choice of test statistics, whether or not deterministic trends are included and/or structural breaks allowed for, the sample of countries, etc.

As for the long-run relationship between healthcare spending and income, a number of papers have used time series approaches for various OECD countries (Blomqvist and Carter 1997; Hansen and King 1998; Gerdtham and Löthgren 2000; MacDonald and Hopkins 2002; Dreger and Reimers 2005) or the US states (Wang and Rettenmaier 2007; Moscone and Tosetti 2010; Freeman 2012). However, the results reported in such studies may not be robust if the underlying data generating process (DGP) for the two series is characterised by structural changes (Freeman 2012). Therefore, some more recent papers allow for structural breaks when testing for cointegration (Jewel et al. 2003; Narayan 2006; Wang and Rettenmaier 2007, among others). On the whole, the evidence is rather mixed. For example, Freeman (2012), using data for the US states over the period 1966-2009, obtains income elasticity estimates below one, while Wang and Rettenmaier (2007) report elasticities higher than one over the period 1980-2000. We revisit these issues using the more sophisticated econometric framework outlined below.

\section{Data and empirical analysis}

We use annual data on healthcare expenditure (HCE) and disposable personal income (DPI) from 1966 to 2009 for 50 US states. The sources for the former are the Centers for Medicare and Medicaid Services Health Expenditures by State of Residence. They report total personal healthcare spending by state and by service, which are expressed in per capita terms. Disposable income is obtained from the US Department of Commerce, Bureau of Economic Analysis. Both HCE and DPI are deflated using the Consumer Price Index. ${ }^{1}$

\subsection{Univariate analysis}

The first step is to estimate the fractional differencing parameter $\mathrm{d}$ in the following setup:

\footnotetext{
${ }^{1}$ We would like to thank Donald G. Freeman, Sam Houston State University, for providing the dataset.
} 


$$
y_{t}=\beta_{0}+\beta_{1} t+x_{t}, \quad(1-L)^{d} x_{t}=u_{t}, \quad t=1,2, \ldots
$$

where $y_{t}$ is the original time series; $\beta_{0}$ and $\beta_{1}$ are the unknown coefficients on the intercept and the linear time trend, respectively; we consider the three standard assumptions of no regressors $\left[\beta_{0}=\beta_{1}=0\right.$ a priori in (1)], an intercept ( $\beta_{0}$ unknown and $\beta_{1}=0$ a priori) and an intercept with a linear trend ( $\beta_{0}$ and $\beta_{1}$ unknown). Specifically, we use a Whittle estimator in the frequency domain as suggested in Dahlhaus (1989).

The results for real disposable personal income for each of the 50 US states are reported in Table 1. In all but one case (Alaska), a linear time trend is required. Concerning the estimates of $d$ (and their corresponding 95\% confidence bands), three groups can be identified, including, respectively:

(a) the states with an order of integration significantly below 1, which indicates mean reversion [Iowa (0.51); Nebraska (0.54), North Dakota (0.66) and South Dakota (0.64)];

(b) those with a value of $d$ significantly above 1 [Alaska (1.27), Hawaii (1.34) and Maryland (1.29)]; and

(c) all the others (the remaining 43), where the unit root null, i.e., $d=1$, cannot be rejected.

Table 2 displays the results for the healthcare expenditure series. A linear time trend is required in all cases and two groups can be identified, including, respectively:

(a) 20 states with $d=1$, namely Alaska, Colorado, Delaware, Hawaii, Idaho, Iowa, Kansas, Maine, Minnesota, Montana, New Mexico, Nebraska, North Dakota, Oklahoma, Oregon, Rhode Island, South Dakota, Washington and Wyoming, and (b) the remaining ones where the estimated value of $d$ is significantly above 1 .

Summary results for both variables are presented in Table 3. Evidence of mean reversion (implying only transitory effects of shocks) is found for Iowa, Nebraska, North Dakota and South Dakota in the case of disposable income.

However, these results could be biased owing to the presence of structural breaks. Given the small number of observations (44), splitting the sample to test for them is not feasible. We follow instead an alternative approach allowing for nonlinearities modelled in the form of Chebyshev polynomials. The model specification is the following:

$$
y_{t}=\sum_{i=0}^{m} \theta_{i} P_{i T}(t)+x_{t}, \quad t=1,2, \ldots,
$$

with $m$ indicating the order of the Chebyshev polynomial, $\theta_{\mathrm{i}}$ denoting the Chebyshev coefficients in time and $x_{t}$ following an $I(d)$ process of the form as in Eq. (1).

The Chebyshev polynomials $P_{i, T}(t)$ in (2) are defined as:

$$
\begin{aligned}
P_{0, T}(t) & =1, \\
P_{i, T}(t) & =\sqrt{2} \cos (i \pi(t-0.5) / T), \quad t=1,2, \ldots, T ; i=1,2, \ldots
\end{aligned}
$$

(see Hamming (1973) and Smyth (1998) for a detailed description of these polynomials). Bierens (1997) uses them in the context of unit root testing. According to 
The relationship between healthcare expenditure and disposable...

Table 1 Estimates of $d$ for each state: real disposable personal income

\begin{tabular}{|c|c|c|c|}
\hline State & No regressors & An intercept & A linear time trend \\
\hline Alabama & $0.91(0.72,1.18)$ & $1.29(0.82,1.67)$ & $1.20(0.95,1.60)$ \\
\hline Alaska & $0.93(0.74,1.19)$ & $1.27(1.06,1.57)$ & $1.25(1.05,1.57)$ \\
\hline Arizona & $0.92(0.73,1.19)$ & $1.31(0.98,1.72)$ & $1.26(0.99,1.70)$ \\
\hline Arkansas & $0.92(0.73,1.19)$ & $1.00(0.66,1.43)$ & $1.01(0.78,1.36)$ \\
\hline California & $0.91(0.72,1.19)$ & $1.10(0.84,1.45)$ & $1.07(0.81,1.44)$ \\
\hline Colorado & $0.90(0.72,1.18)$ & $1.27(0.96,1.64)$ & $1.22(0.93,1.65)$ \\
\hline Connecticut & $0.91(0.72,1.18)$ & $1.01(0.81,1.39)$ & $0.99(0.73,1.36)$ \\
\hline Delaware & $0.91(0.71,1.19)$ & $1.16(0.91,1.49)$ & $1.15(0.86,1.49)$ \\
\hline Florida & $0.92(0.73,1.19)$ & $1.18(0.70,1.56)$ & $1.13(0.89,1.50)$ \\
\hline Georgia & $0.91(0.71,1.19)$ & $1.26(0.92,1.65)$ & $1.21(0.92,1.62)$ \\
\hline Hawaii & $0.93(0.74,1.21)$ & $1.39(1.18,1.67)$ & $1.34(1.15,1.61)$ \\
\hline Idaho & $0.91(0.72,1.19)$ & $1.07(0.83,1.37)$ & $1.05(0.84,1.35)$ \\
\hline Illinois & $0.91(0.71,1.18)$ & $0.90(0.77,1.25)$ & $0.82(0.48,1.24)$ \\
\hline Indiana & $0.91(0.71,1.19)$ & $0.89(0.74,1.26)$ & $0.83(0.52,1.24)$ \\
\hline Iowa & $0.91(0.72,1.19)$ & $0.69(0.60,0.85)$ & $0.51(0.29,0.79)$ \\
\hline Kansas & $0.91(0.72,1.19)$ & $0.96(0.68,1.32)$ & $0.97(0.76,1.27)$ \\
\hline Kentucky & $0.92(0.73,1.21)$ & $0.77(0.65,1.32)$ & $0.91(0.66,1.23)$ \\
\hline Louisiana & $0.92(0.73,1.20)$ & $1.04(0.71,1.44)$ & $1.05(0.85,1.42)$ \\
\hline Maine & $0.91(0.72,1.19)$ & $1.20(0.85,1.59)$ & $1.16(0.85,1.55)$ \\
\hline Maryland & $0.92(0.71,1.19)$ & $1.34(1.04,1.64)$ & $1.29(1.04,1.59)$ \\
\hline Massachusetts & $0.91(0.72,1.19)$ & $1.24(0.96,1.62)$ & $1.25(0.95,1.64)$ \\
\hline Michigan & $0.90(0.71,1.19)$ & $1.12(0.82,1.68)$ & $1.11(0.67,1.68)$ \\
\hline Minnesota & $0.91(0.72,1.18)$ & $0.84(0.71,1.23)$ & $0.79(0.48,1.19)$ \\
\hline Mississippi & $0.92(0.73,1.19)$ & $1.13(0.67,1.51)$ & $1.09(0.87,1.40)$ \\
\hline Missouri & $0.91(0.72,1.19)$ & $0.79(0.69,1.18)$ & $0.67(0.24,1.16)$ \\
\hline Montana & $0.92(0.72,1.18)$ & $0.93(0.74,1.20)$ & $0.92(0.75,1.18)$ \\
\hline New Hampshire & $0.91(0.71,1.19)$ & $1.02(0.84,1.36)$ & $1.01(0.75,1.34)$ \\
\hline New Jersey & $0.91(0.72,1.18)$ & $0.98(0.78,1.36)$ & $0.97(0.69,1.32)$ \\
\hline New Mexico & $0.91(0.72,1.19)$ & $1.08(0.74,1.46)$ & $1.05(0.84,1.39)$ \\
\hline New York & $0.92(0.72,1.19)$ & $1.10(0.86,1.46)$ & $1.10(0.82,1.48)$ \\
\hline Nebraska & $0.91(0.72,1.19)$ & $0.74(0.65,0.89)$ & $0.54(0.29,0.84)$ \\
\hline Nevada & $0.90(0.72,1.17)$ & $1.06(0.76,1.51)$ & $1.03(0.71,1.50)$ \\
\hline N. Carolina & $0.91(0.72,1.19)$ & $1.23(0.89,1.59)$ & $1.17(0.91,1.55)$ \\
\hline N. Dakota & $0.92(0.73,1.19)$ & $0.63(0.47,0.98)$ & $0.66(0.41,0.98)$ \\
\hline Ohio & $0.91(0.72,1.20)$ & $1.06(0.77,1.62)$ & $1.03(0.65,1.59)$ \\
\hline Oklahoma & $0.91(0.73,1.18)$ & $0.87(0.67,1.11)$ & $0.89(0.77,1.08)$ \\
\hline Oregon & $0.91(0.72,1.19)$ & $1.17(0.85,1.57)$ & $1.14(0.85,1.56)$ \\
\hline Pennsylvania & $0.90(0.72,1.18)$ & $1.03(0.73,1.51)$ & $1.03(0.70,1.46)$ \\
\hline Rhode Island & $0.92(0.72,1.20)$ & $1.13(0.88,1.61)$ & $1.15(0.83,1.66)$ \\
\hline
\end{tabular}


Table 1 continued

\begin{tabular}{|c|c|c|c|}
\hline State & No regressors & An intercept & A linear time trend \\
\hline S. Carolina & $0.92(0.72,1.19)$ & $1.27(0.82,1.62)$ & $1.20(0.95,1.54)$ \\
\hline S. Dakota & $0.91(0.72,1.19)$ & $0.71(0.59,0.95)$ & $0.64(0.42,0.94)$ \\
\hline Tennessee & $0.91(0.72,1.19)$ & $1.18(0.82,1.63)$ & $1.12(0.83,1.60)$ \\
\hline Texas & $0.91(0.72,1.19)$ & $0.91(0.71,1.26)$ & $0.93(0.75,1.19)$ \\
\hline Utah & $0.91(0.72,1.18)$ & $1.29(0.93,1.86)$ & $1.28(0.88,1.99)$ \\
\hline Vermont & $0.92(0.72,1.20)$ & $0.89(0.76,1.28)$ & $0.87(0.58,1.26)$ \\
\hline Virginia & $0.92(0.72,1.19)$ & $1.35(0.81,1.76)$ & $1.26(0.94,1.73)$ \\
\hline W. Virginia & $0.92(0.72,1.19)$ & $1.06(0.69,1.45)$ & $1.05(0.82,1.38)$ \\
\hline Washington & $0.91(0.72,1.19)$ & $0.93(0.80,1.29)$ & $0.81(0.50,1.30)$ \\
\hline Wisconsin & $0.92(0.71,1.20)$ & $1.11(0.81,1.53)$ & $1.08(0.79,1.48)$ \\
\hline Wyoming & $0.91(0.73,1.18)$ & $1.15(0.98,1.45)$ & $1.15(0.97,1.47)$ \\
\hline
\end{tabular}

We report in this table the estimates of $d$ in the model given by Eq. (1) along with their 95\% confidence bands for the three cases of no deterministic terms (2nd column), with an intercept ( 3 rd column) and with a linear time trend (4th column). In bold are the significant coefficients according to the deterministic terms. In parentheses, the $95 \%$ confidence band for the estimated values of $d$

Bierens (1997) and Tomasevic and Stanivuk (2009), it is possible to approximate highly nonlinear trends with rather low-degree polynomials. If $m=0$ the model contains an intercept, if $m=1$ it also includes a linear trend, and if $m>1$ it becomes nonlinear-the higher $m$ is the less linear the approximated deterministic component becomes.

The results with $m=3$ are displayed in Table 4 (for disposable income) and in Table 5 (for healthcare expenditure). For disposable income, the estimated value of $d$ is significantly below 1 in five states, namely Iowa, Nebraska, North Dakota, South Dakota and Oklahoma, i.e., the same four as in Table 1 as well as Oklahoma. There are also six cases when $d$ is significantly higher than 1 (in Table 1, this happens in all three cases). More importantly, there is some evidence of nonlinear behaviour in 29 out of the 50 states examined.

The corresponding results for healthcare expenditure are reported in Table 5. There are six states for which the unit root null hypothesis cannot be rejected, namely Alaska, Idaho, Minnesota, Montana, South Dakota and Wyoming. However, for the remaining ones, the estimated value of $d$ insignificantly higher than 1 . Less evidence of nonlinearity is found than for disposable income: significant nonlinear coefficients are only estimated in the cases of Alaska, Minnesota, Nebraska, Nevada, South Dakota and Wyoming. Table 6 summarises the nonlinear results for both variables.

\subsection{Multivariate analysis}

Next, we analyse the long-run relationship between disposable income and healthcare expenditure. Table 7 reports the orders of integration of the two series for each state and provides information on the homogeneity condition. We test for homogeneity in 
Table 2 Estimates of $d$ for each state: healthcare expenditure

\begin{tabular}{|c|c|c|c|}
\hline State & No regressors & An intercept & A linear time trend \\
\hline Alabama & $0.94(0.69,1.24)$ & $1.76(1.43,2.33)$ & $1.49(1.27,1.94)$ \\
\hline Alaska & $0.91(0.69,1.21)$ & $1.08(0.89,1.45)$ & $1.09(0.87,1.42)$ \\
\hline Arizona & $0.96(0.76,1.24)$ & $1.51(0.69,1.83)$ & $1.39(1.19,1.65)$ \\
\hline Arkansas & $0.93(0.69,1.23)$ & $1.48(0.80,1.83)$ & $1.25(1.08,1.51)$ \\
\hline California & $0.96(0.76,1.24)$ & $1.62(1.35,2.12)$ & $1.45(1.23,1.87)$ \\
\hline Colorado & $0.93(0.72,1.22)$ & $1.28(0.77,1.64)$ & $1.16(0.92,1.47)$ \\
\hline Connecticut & $0.94(0.71,1.24)$ & $1.54(1.21,1.95)$ & $1.39(1.15,1.75)$ \\
\hline Delaware & $0.92(0.70,1.22)$ & $1.43(0.87,1.85)$ & $1.25(0.98,1.58)$ \\
\hline Florida & $0.95(0.73,1.25)$ & $1.72(1.44,2.22)$ & $1.45(1.26,1.80)$ \\
\hline Georgia & $0.95(0.72,1.25)$ & $1.74(1.47,2.20)$ & $1.51(1.32,1.83)$ \\
\hline Hawaii & $0.95(0.74,1.23)$ & $0.90(0.67,1.76)$ & $1.10(0.96,1.51)$ \\
\hline Idaho & $0.93(0.70,1.23)$ & $1.12(0.81,1.61)$ & $1.09(0.80,1.49)$ \\
\hline Illinois & $0.93(0.72,1.22)$ & $1.53(0.75,2.01)$ & $1.28(1.06,1.62)$ \\
\hline Indiana & $0.93(0.69,1.22)$ & $1.58(1.16,2.14)$ & $1.38(1.08,1.82)$ \\
\hline Iowa & $0.92(0.69,1.22)$ & $1.22(0.80,1.76)$ & $1.00(0.82,1.50)$ \\
\hline Kansas & $0.94(0.72,1.23)$ & $1.41(0.74,1.86)$ & $1.21(0.93,1.63)$ \\
\hline Kentucky & $0.92(0.65,1.24)$ & $1.69(1.32,2.32)$ & $1.42(1.18,1.82)$ \\
\hline Louisiana & $0.95(0.72,1.24)$ & $1.63(1.38,2.03)$ & $1.39(1.21,1.74)$ \\
\hline Maine & $0.93(0.70,1.23)$ & $1.48(0.88,1.97)$ & $1.29(0.99,1.68)$ \\
\hline Maryland & $0.95(0.74,1.24)$ & $1.64(1.37,2.06)$ & $1.42(1.20,1.75)$ \\
\hline Massachusetts & $0.94(0.74,1.23)$ & $1.65(1.28,2.22)$ & $1.47(1.17,1.94)$ \\
\hline Michigan & $0.94(0.72,1.22)$ & $1.55(1.24,2.04)$ & $1.28(1.08,1.62)$ \\
\hline Minnesota & $0.92(0.59,1.22)$ & $1.12(0.79,1.56)$ & $1.05(0.85,1.34)$ \\
\hline Mississippi & $0.93(0.57,1.24)$ & $1.39(0.79,1.72)$ & $1.19(1.01,1.46)$ \\
\hline Missouri & $0.95(0.73,1.24)$ & $1.42(0.69,1.83)$ & $1.21(0.98,1.54)$ \\
\hline Montana & $0.93(0.71,1.23)$ & $0.89(0.77,1.61)$ & $1.01(0.71,1.41)$ \\
\hline New Hampshire & $0.93(0.69,1.22)$ & $1.44(1.05,1.88)$ & $1.35(1.09,1.73)$ \\
\hline New Jersey & $0.92(0.68,1.22)$ & $1.63(1.36,2.02)$ & $1.49(1.26,1.82)$ \\
\hline New Mexico & $0.94(0.71,1.24)$ & $0.89(0.77,1.63)$ & $1.05(0.77,1.44)$ \\
\hline New York & $0.94(0.72,1.24)$ & $1.79(1.42,2.44)$ & $1.56(1.28,1.94)$ \\
\hline Nebraska & $0.93(0.71,1.22)$ & $1.42(0.78,1.85)$ & $1.22(0.96,1.59)$ \\
\hline Nevada & $0.96(0.76,1.24)$ & $1.39(1.15,1.71)$ & $1.27(1.10,1.51)$ \\
\hline N. Carolina & $0.93(0.67,1.24)$ & $1.55(1.24,1.95)$ & $1.36(1.14,1.66)$ \\
\hline N. Dakota & $0.95(0.73,1.25)$ & $1.41(1.04,1.86)$ & $1.25(0.98,1.67)$ \\
\hline Ohio & $0.92(0.69,1.23)$ & $1.66(1.30,2.30)$ & $1.38(1.12,1.89)$ \\
\hline Oklahoma & $0.95(0.73,1.24)$ & $1.17(0.72,1.67)$ & $1.08(0.79,1.43)$ \\
\hline Oregon & $0.95(0.74,1.24)$ & $1.32(0.84,1.82)$ & $1.16(0.87,1.54)$ \\
\hline Pennsylvania & $0.92(0.69,1.23)$ & $1.58(1.30,1.98)$ & $1.35(1.15,1.66)$ \\
\hline Rhode Island & $0.93(0.71,1.22)$ & $1.35(0.79,1.77)$ & $1.19(0.88,1.58)$ \\
\hline S. Carolina & $0.93(0.66,1.24)$ & $1.61(1.32,1.94)$ & $1.40(1.19,1.72)$ \\
\hline
\end{tabular}


Table 2 continued

\begin{tabular}{|c|c|c|c|}
\hline State & No regressors & An intercept & A linear time trend \\
\hline S. Dakota & $0.92(0.69,1.23)$ & $0.85(0.78,1.71)$ & $0.89(0.62,1.33)$ \\
\hline Tennessee & $0.92(0.68,1.22)$ & $1.45(1.21,1.81)$ & $1.26(1.10,1.49)$ \\
\hline Texas & $0.95(0.73,1.24)$ & $1.57(1.27,1.93)$ & $1.29(1.10,1.59)$ \\
\hline Utah & $0.93(0.70,1.23)$ & $1.51(1.03,1.97)$ & $1.33(1.04,1.76)$ \\
\hline Vermont & $0.92(0.70,1.22)$ & $1.39(1.06,1.81)$ & $1.36(1.11,1.74)$ \\
\hline Virginia & $0.95(0.72,1.24)$ & $1.59(1.32,1.80)$ & $1.33(1.14,1.64)$ \\
\hline W. Virginia & $0.93(0.68,1.24)$ & $1.56(1.22,2.03)$ & $1.33(1.11,1.62)$ \\
\hline Washington & $0.94(0.73,1.24)$ & $1.44(0.75,1.89)$ & $1.25(0.91,1.64)$ \\
\hline Wisconsin & $0.93(0.73,1.23)$ & $1.44(1.07,1.93)$ & $1.25(1.02,1.51)$ \\
\hline Wyoming & $0.92(0.69,1.21)$ & $1.04(0.93,1.28)$ & $1.06(0.91,1.32)$ \\
\hline
\end{tabular}

We report in this table the estimates of $d$ in the model given by Eq. (1) along with their 95\% confidence bands for the three cases of no deterministic terms (2nd column), with an intercept (3rd column) and with a linear time trend (4rd column). In bold are the significant coefficients according to the deterministic terms. In parentheses, the $95 \%$ confidence band for the estimated values of $d$

Table 3 Grouping of the states according to the degrees of integration

\begin{tabular}{lll}
\hline$d$ & Disposable personal income & HC expenditure \\
\hline$d<1$ & Iowa, Nebraska, North Dakota, South Dakota & \\
$d=1$ & Alabama, Arizona Arkansas, California, & Alaska, Colorado, Delaware, Hawaii, \\
Colorado, Connecticut, Delaware, Florida, & Idaho, Iowa, Kansas, Maine, \\
& Georgia, Idaho, Illinois, Indiana, Kansas, & Minnesota, Missouri, Montana, \\
& Kentucky, Louisiana, Maine, Massachusetts, & New Mexico, Nebraska, North \\
Michigan, Minnesota, Mississippi, Missouri, & Dakota, Oklahoma, Oregon, Rhode \\
Montana, New Hampshire, New Jersey, New & Island, South Dakota, Washington, \\
& Mexico, New York, Nevada, North Caroline, & Wyoming \\
& Ohio, Oklahoma, Oregon, Pennsylvania, & \\
Rhode Island, South Caroline, Tennessee, & \\
Texas, Utah, Vermont, Virginia, West Virginia, & \\
Washington, Wisconsin, Wyoming & \\
Alaska, Hawaii, Maryland & Alabama, Arizona, Arkansas, \\
& California, Connecticut, Florida, \\
& Georgia, Illinois, Indiana, \\
& Kentucky, Louisiana, Maryland, \\
& & Massachusetts, Michigan, \\
& & Mississippi, New Hampshire, New
\end{tabular}

$d$ refers to the estimates of the fractional differencing parameter in Eq. (1)

the order of integration of the variables by using an adaptation of Robinson and Yajima (2002) statistic $\hat{T}_{x y}$ to log-periodogram estimation (Robinson 1995b). The statistic is the following: 
The relationship between healthcare expenditure and disposable...

Table 4 Estimates of $d$ based on a nonlinear model for disposable personal income

\begin{tabular}{|c|c|c|c|c|c|}
\hline State & ncome & $\theta_{0}$ & $\theta_{1}$ & $\theta_{2}$ & $\theta_{3}$ \\
\hline Alabama & $1.30(1.05,1.59)$ & 4.443 (26.57) & $-0.222(-2.09)$ & $-0.008(-0.22)$ & $(-1.42)$ \\
\hline Alaska & $0.98(0.60,1.43)$ & $4.969(39.22)$ & $-0.091(-1.24)$ & $-0.041(-1.10)$ & $-0.094(-3.74)$ \\
\hline Arizona & $28(0.97,1.62)$ & $4.561(23.82)$ & $-0.172(-1.42)$ & $0.007(0.17)$ & $-0.047(-1.78)$ \\
\hline Arkansas & $1.05(0.78,1.40)$ & $4.451(39.24)$ & $-0.223(-3.32)$ & $-0.011(-0.37)$ & $-0.045(-2.20)$ \\
\hline California & $0.96(0.62,1.35)$ & $4.882(86.48)$ & $-0.150(-4.62)$ & $-0.021(-0.12)$ & $-0.038(-3.29)$ \\
\hline Colorado & $12(0.84,1.45)$ & $4.778(52.32)$ & $-0.226(-4.10)$ & $0.001(0.04)$ & $-0.043(-2.84)$ \\
\hline Connecticut & $1.05(0.7)$ & $4.967(48.86)$ & $-0.231(-3.85)$ & $-0.014(-0.50)$ & $-0.015(-0.81)$ \\
\hline Delaware & $1.13(0.85,1.46)$ & $4.801(42.21)$ & $-0.176(-2.56)$ & $-0.001(-0.06)$ & $-0.009(-0.48)$ \\
\hline Florida & $1.21(0.94,1.58)$ & $4.633(28.23)$ & $-0.194(-1.91)$ & $-0.009(-0.22)$ & $-0.031(-1.29)$ \\
\hline Georgia & $1.24(0.93,1.57)$ & $4.549(27.95)$ & $-0.228(-2.25)$ & $-0.005(-0.14)$ & $-0.012(-0.54)$ \\
\hline Hawaii & 1.60) & $(18.51)$ & $-0.039(-0.24)$ & $-0.002(-0.05)$ & $-0.009(-0.33)$ \\
\hline Idaho & $1.07(0.87,1.34)$ & $4.524(38.60)$ & $-0.170(-2.44)$ & $-0.016(-0.49)$ & $-0.031(-1.70)$ \\
\hline Illinois & 2) & 4.855 (96.16) & $-0.179(-6.28)$ & 0.002 & $-0.020(-1.77)$ \\
\hline Indiana & 0 & 9) & $-0.173(-5.50)$ & -0.001 & $-0.015(-1.13)$ \\
\hline Iowa & $0.77)$ & $4.744(226.69)$ & $-0.176(-13.36)$ & $0.009(0.83)$ & $-0.038(-4.10)$ \\
\hline Kansas & $0.98(0.72,1.30)$ & $4.706(59.23)$ & $-0.190(-4.13)$ & $-0.007(-0.30)$ & $-0.043(-2.76)$ \\
\hline Kentucky & $0.99(0.68,1.33)$ & 4.503 (54.89) & $-0.207(-4.35)$ & $-0.008(-0.34)$ & $-0.031(-1.95)$ \\
\hline iana & ) & 1) & $-0.225(-5.75)$ & $-0.002(-0.10)$ & $-0.058(-4.29)$ \\
\hline Maine & $23(0.94,1.59)$ & $4.583(30.09)$ & $-0.209(-2.20)$ & $-0.001(-0.03)$ & $-0.020(-0.93)$ \\
\hline Maryland & $1.37(1.14,1.61)$ & $4.733(22.84)$ & $-0.185(-1.39)$ & $-0.003(-0.08)$ & $-0.029(-1.11)$ \\
\hline Massach & 1.2 & $12(2 / .01)$ & $-0.228(-2.03)$ & $0.007(0.17)$ & $-0.011(-0.46)$ \\
\hline Michigan & $1.02(0.57,1.56)$ & $5(48.39)$ & $-0.147(-2.54)$ & $-0.001(-0.04)$ & $-0.007(-0.40)$ \\
\hline Minnesota & $0.83(0.50,1.20)$ & 4.777 (77.52) & $-0.222(-6.44)$ & $-0.02(-0.09)$ & $-0.032(-2.13)$ \\
\hline Mississippi & 54) & $4.291(28.27)$ & $-0.223(-2.39)$ & $0.005(0.14)$ & $-0.039(-1.67)$ \\
\hline Missouri & $0.76(0.30,1.21)$ & $4.718(115.75)$ & $-0.194(-8.54)$ & $-0.004(-0.28)$ & $-0.024(-2.22)$ \\
\hline Montana & $79(0.56,1.08)$ & $4.05)$ & $-0.163(-5.33)$ & $0.013(0.72)$ & $-0.056(-4.03)$ \\
\hline hire & $0.95(0.63,1.30)$ & $4.813(61.62)$ & $-0.256(-5.71)$ & $-0.018(-0.78)$ & $-0.019(-1.23)$ \\
\hline New Jersey & $1.07(0.82,1.38)$ & $04(51.03)$ & $-0.219(-3.85)$ & $-0.004(-0.18)$ & $-0.014(-0.86)$ \\
\hline New Mexico & $0.97(0.66,1.35)$ & $52(75.44)$ & $-0.194(-5.56)$ & $-0.006(-0.37)$ & $-0.050(-4.11)$ \\
\hline$\Delta$ & 80. & $45(51.10)$ & $-0.1 / 7(-2.22)$ & $0.020(0.00)$ & $-0.005(-0.23)$ \\
\hline Nebraska & $90)$ & 75 (148.82) & $-0.206(-11.30)$ & $0.004(0.29)$ & $-0.034(-3.06)$ \\
\hline Nevada & $1.05(0.74,1.44)$ & $4.795(45.62)$ & $-0.139(-2.24)$ & $0.006(0.22)$ & $-0.025(-1.32)$ \\
\hline N. Carolina & $1.22(0.94,1.61)$ & $21(29.21)$ & $-0.231(-2.41)$ & $-0.011(-0.31)$ & $-0.01(-0.47)$ \\
\hline kota & ) & $77(63.66)$ & $-0.202(-4.76)$ & $0.017(0.53)$ & $-0.074(-2.79)$ \\
\hline Ohio & $1.05(0.66,1.56)$ & $4.723(57.14)$ & $-0.162(-3.31)$ & $-0.005(-0.23)$ & $-0.016(-1.08)$ \\
\hline Oklahoma & $0.39(-0.01,0.77)$ & $4.663(390.38)$ & $-0.186(-24.10)$ & $-0.01(-1.65)$ & $-0.067(-11.77)$ \\
\hline Oregon & $1.16(0.88,1.52)$ & $4.669(38.73)$ & $-0.172(-2.34)$ & $0.006(0.21)$ & $-0.027(-1.66)$ \\
\hline Pennsylvania & $1.16(0.86,1.52)$ & 4.705 & $-0.184(-2.96)$ & $-0.002(-0.07)$ & $-0.021(-1.30)$ \\
\hline Rhode Island & $1.19(0.88,1.55)$ & $4.738(38.36)$ & $-0.193(-2.53)$ & $0.005(0.16)$ & $-0.012(-0.64)$ \\
\hline S. Carolina & $1.30(1.05,1.57)$ & $4.415(25.85)$ & $-0.215(-1.98)$ & $-0.003(-0.09)$ & $-0.020(-0.89)$ \\
\hline
\end{tabular}


Table 4 continued

\begin{tabular}{|c|c|c|c|c|c|}
\hline State & Income & $\theta_{0}$ & $\theta_{1}$ & $\theta_{2}$ & $\theta_{3}$ \\
\hline S. Dakota & $0.61(0.32,0.94)$ & $4.673(84.04)$ & $-0.234(-7.42)$ & $0.017(0.76)$ & $-0.052(-2.80)$ \\
\hline Tennessee & $1.17(0.87,1.60)$ & $4.548(33.56)$ & $-0.237(-2.86)$ & $0.01(-0.31)$ & $-0.020(-0.94)$ \\
\hline Texas & $0.74(0.39,1.09)$ & $4.705(140.63)$ & $-0.215(-11.58)$ & $-0.016(-1.32)$ & $-0.052(-5.70)$ \\
\hline Utah & $1.13(0.71,1.62)$ & $4.576(47.19)$ & $-0.179(-3.05)$ & $0.014(0.55)$ & $-0.043(-2.69)$ \\
\hline Vermont & $0.98(0.65,1.32)$ & $4.5547(59.88)$ & $-0.231(-5.12)$ & $0.009(0.38)$ & $-0.024(-1.68)$ \\
\hline Virginia & $1.34(1.03,1.58)$ & $4.664(27.16)$ & $-0.218(-1.99)$ & $-0.012(-0.31)$ & $-0.035(-1.68)$ \\
\hline W. Virginia & $1.11(0.85,1.44)$ & $4.462(42.76)$ & $-0.185(-2.94)$ & $0.004(0.02)$ & $-0.035(-1.99)$ \\
\hline Washington & $0.73(0.30,1.22)$ & $4.853(168.69)$ & $-0.199(-12.42)$ & $0.008(0.008)$ & $-0.032(-4.01)$ \\
\hline Wisconsin & $1.07(0.78,1.46)$ & $4.698(57.20)$ & $-0.184(-3.76)$ & $0.004(0.19)$ & $-0.031(-2.11)$ \\
\hline Wyoming & $0.80(0.53,1.16)$ & $4.782(82.06)$ & $-0.200(-6.17)$ & $0.023(1.14)$ & $-0.096(-6.53)$ \\
\hline
\end{tabular}

The 2nd column refers to the estimates of $d$ (and their associated 95\% confidence intervals in the model given by Eq. (2) with $I(d) x_{t}$. The values in columns 3, 4, 5 and 6 refers to the Chebyshev coefficients in Eq. (2) $(t$ values in parenthesis). In bold are significant coefficients at the $5 \%$ level

$$
\hat{T}_{x y}=\frac{m^{1 / 2}\left(\hat{d}_{x}-\hat{d}_{y}\right)}{\left(\frac{1}{2}\left(1-\hat{G}_{x y} /\left(\hat{G}_{x x} \hat{G}_{y y}\right)\right)^{1 / 2}+h(n)\right.}
$$

where $m$ is a bandwidth parameter, $d_{x}$ and $d_{y}$ are the orders of integration of each of the series, $I\left(\lambda_{j}\right)$ is the cross-periodogram in the bivariate representation of the series, $\mathrm{h}(\mathrm{n})>0$ and $\hat{G}_{x y}$ is the $(x y)$ th element of

$$
\begin{gathered}
\hat{G}=\frac{1}{m} \sum_{j=1}^{m} \operatorname{Re}\left[\hat{\Lambda}\left(\lambda_{j}\right)^{-1} I\left(\lambda_{j}\right) \hat{\Lambda}\left(\lambda_{j}\right)^{-1 *}\right], \\
\hat{\Lambda}\left(\lambda_{j}\right)=\operatorname{diag}\left\{\mathrm{e}^{i \pi \hat{d}_{x} / 2} \lambda^{-\hat{d}_{x}}, \mathrm{e}^{i \pi \hat{d}_{y} / 2} \lambda^{-\hat{d}_{y}}\right\},
\end{gathered}
$$

with a standard normal limit distribution (see Gil-Alana and Hualde 2009, for evidence on the finite sample performance of this procedure). This is satisfied in all cases with the exception of Iowa, Nebraska and North Dakota, where the orders of integration for disposable income $(0.51,0.54$ and 0.66 , respectively) are much lower than for healthcare expenditure $(1.00,1.22$ and 1.25 , respectively). Therefore, cointegration between the two series can be ruled out in these three cases. For the remaining states, we test for cointegration using a two-step method, similar in spirit to the one proposed by Engle and Granger (1987): first, we regress healthcare expenditure on disposable income, and then, in the second step, we test the order of integration of the estimated residuals. This approach is followed, for instance, in Gil-Alana (2003). Specifically, we first run the regression:

$$
\log (\mathrm{HEALTH})_{t}=\beta_{0}+\beta_{1} \log (\mathrm{INCOME})_{t}+x_{t}, \quad t=1,2, \ldots
$$

and then the fractional differencing parameter $d$ is estimated for the residuals from the above equation. 
The relationship between healthcare expenditure and disposable...

Table 5 Estimates of $d$ based on a nonlinear model for healthcare expenditure

\begin{tabular}{|c|c|c|c|c|c|}
\hline State & Health & $\theta_{0}$ & $\theta_{1}$ & $\theta_{2}$ & $\theta_{3}$ \\
\hline Alabama & $1.68(1.40,1.74)$ & $1.907(3.23)$ & $-0.277(-0.70)$ & $-0.072(-0.65)$ & $0.060(-1.08)$ \\
\hline Alaska & $0.82(0.53,1.27)$ & $2.836(42.63)$ & $-0.523(-14.04)$ & $-0.005(-0.23)$ & $-0.098(-5.99)$ \\
\hline Arizona & $1.49(1.29,1.71)$ & $2.109(5.49)$ & $-0.195(-0.77)$ & $-0.048(-0.61)$ & $-0.020(-0.48)$ \\
\hline Arkansas & $1.45(1.22,1.73)$ & $2.097(6.65)$ & $-0.390(-1.89)$ & $-0.048(-0.74)$ & $-0.025(-0.70)$ \\
\hline California & $1.56(1.32,1.72)$ & $2.202(5.41)$ & $-0.068(-0.25)$ & $-0.054(-0.68)$ & $-0.034(-0.81)$ \\
\hline Colorado & $1.35(1.12,1.57)$ & $2.429(11.36)$ & $-0.300(-2.19)$ & $-0.021(-0.45)$ & $-0.019(-0.71)$ \\
\hline Connecticut & $1.54(1.29,1.70)$ & $2.276(5.27)$ & $-0.236(-0.83)$ & $-0.026(-0.30)$ & $0.023(0.50)$ \\
\hline Delaware & $1.47(1.24,1.71)$ & $2.361(7.49)$ & $-0.352(-1.71)$ & $-0.014(-0.23)$ & $-0.009(-0.25)$ \\
\hline Florida & $1.61(1.38,1.73)$ & $2.061(4.43)$ & $-0.161(-0.52)$ & $-0.080(-0.90)$ & $0.002(0.05)$ \\
\hline Georgia & $1.61(1.40,1.74)$ & $1.933(3.96)$ & $-0.229(-0.70)$ & $-0.070(-0.75)$ & $0.002(0.05)$ \\
\hline Hawaii & $1.38(1.14,1.67)$ & $2.287(7.45)$ & $-0.277(-1.40)$ & $-0.039(-0.59)$ & $-0.010(-0.27)$ \\
\hline Idaho & $1.24(0.96,1.57)$ & $2.164(7.88)$ & $-0.477(-2.61)$ & $0.016(0.25)$ & $-0.020(-0.51)$ \\
\hline Illinois & $1.51(1.29,1.68)$ & $2.305(7.33)$ & $-0.262(-1.26)$ & $-0.029(-0.46)$ & $-0.018(-0.53)$ \\
\hline Indiana & $1.56(1.28,1.70)$ & $2.144(5.08)$ & $-0.282(-1.00)$ & $-0.024(-0.29)$ & $-0.011(-0.24)$ \\
\hline Iowa & $1.31(1.02,1.64)$ & $2.416(10.21)$ & $-0.415(-2.76)$ & $-0.005(-0.10)$ & $-0.028(-0.90)$ \\
\hline Kansas & $1.45(1.17,1.76)$ & $2.266(6.75)$ & $-0.326(-1.67)$ & $-0.010(-0.15)$ & $-0.023(-0.60)$ \\
\hline Kentucky & $1.57(1.31,1.72)$ & $2.031(5.47)$ & $-0.336(-1.37)$ & $-0.023(-0.32)$ & $0.001(0.02)$ \\
\hline Louisiana & $1.57(1.37,1.70)$ & $2.01(4.82)$ & $-0.264(-0.95)$ & $-0.063(-0.77)$ & $-0.015(-0.34)$ \\
\hline Maine & $1.54(1.26,1.72)$ & $2.141(5.25)$ & $-0.361(-1.34)$ & $0.025(0.30)$ & $-0.024(-0.55)$ \\
\hline Maryland & $1.57(1.40,1.73)$ & $2.095(5.05)$ & $-0.207(-0.75)$ & $-0.034(-0.42)$ & $-0.021(-0.50)$ \\
\hline Massachusetts & $1.59(1.38,1.72)$ & $2.307(5.10)$ & $-0.185(-0.61)$ & $-0.031(-0.36)$ & $0.0008(-0.01)$ \\
\hline Michigan & $1.48(1.25,1.70)$ & $2.319(8.12)$ & $-0.255(-1.36)$ & $-0.049(-0.85)$ & $-0.025(-0.79)$ \\
\hline Minnesota & $1.13(0.81,1.47)$ & $2.661(2.45)$ & $-0.463(-5.88)$ & $-0.031(-0.94)$ & $-0.053(-2.51)$ \\
\hline Mississippi & $1.39(1.16,1.68)$ & $2.041(6.09)$ & $-0.492(-2.27)$ & $-0.031(-0.43)$ & $-0.048(-1.18)$ \\
\hline Missouri & $1.38(1.08,1.70)$ & $2.369(8.43)$ & $-0.328(-1.81)$ & $-0.045(-0.75)$ & $-0.041(-1.20)$ \\
\hline Montana & $1.26(0.98,1.57)$ & $2.335(9.99)$ & $-0.437(-2.98)$ & $-0.003(-0.05)$ & $-0.035(-1.06)$ \\
\hline New Hampshire & $1.48(1.29,1.71)$ & $2.234(6.81)$ & $-0.337(-1.76)$ & $-0.012(-0.18)$ & $0.006(-0.02)$ \\
\hline New Jersey & $1.56(1.35,1.72)$ & $2.231(5.54)$ & $-0.318(-1.19)$ & $-0.035(-0.44)$ & $-0.012(-0.28)$ \\
\hline New Mexico & $1.32(1.10,1.57)$ & $2.187(8.27)$ & $-0.412(-2.45)$ & $-0.019(-0.33)$ & $-0.019(-0.55)$ \\
\hline New York & $1.61(1.48,1.72)$ & $2.244(5.25)$ & $-0.132(-0.46)$ & $-0.014(-0.17)$ & $0.023(0.53)$ \\
\hline Nebraska & $1.42(1.15,1.72)$ & $2.359(8.80)$ & $-0.379(-2.18)$ & $-0.013(-0.23)$ & $-0.055(-1.72)$ \\
\hline Nevada & $1.29(1.01,1.57)$ & $2.381(9.57)$ & $-0.311(-1.98)$ & $-0.049(-0.87)$ & $-0.072(-2.14)$ \\
\hline N. Carolina & $1.55(1.29,1.71)$ & $1.968(4.50)$ & $-0.363(-1.25)$ & $-0.015(-0.17)$ & $-0.005(-0.11)$ \\
\hline N. Dakota & $1.38(1.04,1.70)$ & $2.393(6.67)$ & $-0.351(-1.72)$ & $-0.04(-0.51)$ & $-0.047(-1.07)$ \\
\hline Ohio & $1.56(1.24,1.70)$ & $2.286(6.04)$ & $-0.282(-1.12)$ & $-0.053(-0.71)$ & $-0.030(-0.76)$ \\
\hline Oklahoma & $1.36(1.13,1.68)$ & $2.211(7.64)$ & $-0.342(-1.84)$ & $-0.001(-0.16)$ & $-0.022(-0.61)$ \\
\hline Oregon & $1.39(1.11,1.71)$ & 2.267 (7.82) & $-0.305(-1.86)$ & $-0.005(-0.08)$ & $-0.040(-1.12)$ \\
\hline Pennsylvania & $1.49(1.26,1.74)$ & $2.384(8.00)$ & $-0.347(-1.77)$ & $-0.055(-0.91)$ & $-0.011(-0.33)$ \\
\hline Rhode Island & $1.41(1.18,1.72)$ & $2.499(8.82)$ & $-0.358(-1.95)$ & $-0.010(-0.17)$ & $-0.027(-0.82)$ \\
\hline S. Carolina & $1.57(1.34,1.72)$ & $1.871(4.16)$ & $-0.377(-1.26)$ & $-0.018(-0.21)$ & $-0.006(-0.14)$ \\
\hline
\end{tabular}


Table 5 continued

\begin{tabular}{|c|c|c|c|c|c|}
\hline State & Health & $\theta_{0}$ & $\theta_{1}$ & $\theta_{2}$ & $\theta_{3}$ \\
\hline S. Dakota & $1.24(0.91,1.57)$ & $2.428(13.14)$ & $-0.466(-4.04)$ & $-0.010(-0.23)$ & $-0.038(-1.65)$ \\
\hline Tennessee & $1.35(1.09,1.63)$ & $2.367(9.41)$ & $-0.466(-4.04)$ & $-0.060(-1.08)$ & $-0.032(-1.01)$ \\
\hline Texas & $1.52(1.28,1.71)$ & $2.087(6.27)$ & $-0.447(-2.78)$ & $-0.032(-0.49)$ & $-0.021(-0.58)$ \\
\hline Utah & $1.48(1.21,1.70)$ & $2.074(6.36)$ & $-0.246(-1.12)$ & $-0.027(-0.41)$ & $-0.026(-0.72)$ \\
\hline Vermont & $1.44(1.15,1.74)$ & $2.265(6.83)$ & $-0.399(-1.85)$ & $0.064(-0.93)$ & $-0.013(-0.34)$ \\
\hline Virginia & $1.56(1.31,1.75)$ & $2.003(5.14)$ & $-0.263(-1.02)$ & $-0.055(-0.72)$ & $-0.032(-0.80)$ \\
\hline W. Virginia & $1.59(1.31,1.74)$ & $2.009(4.39)$ & $-0.301(-0.99)$ & $-0.032(-0.36)$ & $0.011(-0.23)$ \\
\hline Washington & $1.47(1.23,1.71)$ & $2.257(6.98)$ & $-0.228(-1.08)$ & $-0.026(-0.39)$ & $-0.013(-0.36)$ \\
\hline Wisconsin & $1.47(1.23,1.72)$ & $2.292(7.15)$ & $-0.350(-1.68)$ & $0.0001(0.01)$ & $-0.040(-1.11)$ \\
\hline Wyoming & $0.90(0.21,1.28)$ & 2.494 (23.09) & $-0.524(-10.40)$ & $0.064(2.27)$ & $-0.009(-0.46)$ \\
\hline
\end{tabular}

The 2nd column refers to the estimates of $d$ (and their associated 95\% confidence intervals in the model given by Eq. (2) with $I(d) x_{t}$. The values in columns 3, 4, 5 and 6 refers to the Chebyshev coefficients in Eq. (2) $(t$ values in parenthesis). In bold are significant coefficients at the $5 \%$ level

Table 6 Summary of the nonlinear results

\begin{tabular}{|c|c|c|}
\hline & Disposable personal income & Healthcare expenditure \\
\hline Linear & $\begin{array}{l}\text { Alabama, Connecticut, Delaware, } \\
\text { Florida, Georgia, Hawaii, Indiana, } \\
\text { Maine, Maryland, Massachusetts, } \\
\text { Michigan, New Hampshire, New } \\
\text { Jersey, New York. Nevada, North } \\
\text { Carolina, Ohio, Pennsylvania, } \\
\text { Rhode Island, South Carolina, } \\
\text { Tennessee }\end{array}$ & $\begin{array}{l}\text { Alabama, Arizona, Arkansas, } \\
\text { California, Colorado, Connecticut, } \\
\text { Delaware, Florida, Georgia, } \\
\text { Hawaii, Idaho, Illinois, Indiana, } \\
\text { Iowa, Kansas, Kentucky, Louisiana, } \\
\text { Maine, Maryland, Massachusetts, } \\
\text { Michigan, Mississippi, Missouri, } \\
\text { Montana, New Hampshire, New } \\
\text { Jersey, New Mexico, New York, } \\
\text { North Carolina, North Dakota, } \\
\text { Ohio, Oklahoma, Oregon, } \\
\text { Pennsylvania, Rhode Island, South } \\
\text { Carolina, Tennessee, Utah, } \\
\text { Vermont, Virginia, West Virginia, } \\
\text { Washington, Wisconsin }\end{array}$ \\
\hline Nonlinear & $\begin{array}{l}\text { Alaska, Arizona, Arkansas, } \\
\text { California, Colorado, Idaho, } \\
\text { Illinois, Iowa, Kansas, Kentucky, } \\
\text { Louisiana, Minnesota, Mississippi, } \\
\text { Missouri, Montana, New Mexico, } \\
\text { Nebraska, North Dakota, } \\
\text { Oklahoma, Oregon, South Dakota, } \\
\text { Texas, Utah, Vermont, Virginia, } \\
\text { West Virginia, Washington, } \\
\text { Wisconsin, Wyoming }\end{array}$ & $\begin{array}{l}\text { Alaska, Minnesota, Nebraska, } \\
\text { Nevada, South Dakota, Wyoming }\end{array}$ \\
\hline
\end{tabular}

Table 8 reports the estimates of $\beta_{1}$ and those of $d$ based on both parametric and semiparametric methods, in the latter case using three different bandwidth parameters, $T^{0.4}, T^{0.5}$ and $T^{0.4}$. The $\beta_{1}$ coefficients are all statistically significant, and 
The relationship between healthcare expenditure and disposable...

Table 7 Estimates of $d$ for each state and homogeneity in the value of $d$

\begin{tabular}{|c|c|c|c|}
\hline State & DPI & $\mathrm{HCE}$ & Homogeneity \\
\hline Alabama & $1.20(0.95,1.60)$ & $1.49(1.27,1.94)$ & $\gamma$ \\
\hline Alaska & $1.27(1.06,1.57)$ & $1.09(0.87,1.42)$ & $\gamma$ \\
\hline Arizona & $1.26(0.99,1.70)$ & $1.39(1.19,1.65)$ & $\gamma$ \\
\hline Arkansas & $1.01(0.78,1.36)$ & $1.25(1.08,1.51)$ & $\gamma$ \\
\hline California & $1.07(0.81,1.44)$ & $1.45(1.23,1.87)$ & $\gamma$ \\
\hline Colorado & $1.22(0.93,1.65)$ & $1.16(0.92,1.47)$ & $\gamma$ \\
\hline Connecticut & $0.99(0.73,1.36)$ & $1.39(1.15,1.75)$ & $\gamma$ \\
\hline Delaware & $1.15(0.86,1.49)$ & $1.25(0.98,1.58)$ & $\gamma$ \\
\hline Florida & $1.13(0.89,1.50)$ & $1.45(1.26,1.80)$ & $\gamma$ \\
\hline Georgia & $1.21(0.92,1.62)$ & $1.51(1.32,1.83)$ & $\gamma$ \\
\hline Hawaii & $1.34(1.15,1.61)$ & $1.10(0.96,1.51)$ & $\gamma$ \\
\hline Idaho & $1.05(0.84,1.35)$ & $1.09(0.80,1.49)$ & $\gamma$ \\
\hline Illinois & $0.82(0.48,1.24)$ & $1.28(1.06,1.62)$ & $\gamma$ \\
\hline Indiana & $0.83(0.52,1.24)$ & $1.38(1.08,1.82)$ & $\gamma$ \\
\hline Iowa & $0.51(0.29,0.79)$ & $1.00(0.82,1.50)$ & NO HOMOG. \\
\hline Kansas & $0.97(0.76,1.27)$ & $1.21(0.93,1.63)$ & $\gamma$ \\
\hline Kentucky & $0.91(0.66,1.23)$ & $1.42(1.18,1.82)$ & $\gamma$ \\
\hline Louisiana & $1.05(0.85,1.42)$ & $1.39(1.21,1.74)$ & $\gamma$ \\
\hline Maine & $1.16(0.85,1.55)$ & $1.29(0.99,1.68)$ & $\gamma$ \\
\hline Maryland & $1.29(1.04,1.59)$ & $1.42(1.20,1.75)$ & $\gamma$ \\
\hline Massachusetts & $1.25(0.95,1.64)$ & $1.47(1.17,1.94)$ & $\gamma$ \\
\hline Michigan & $1.11(0.67,1.68)$ & $1.28(1.08,1.62)$ & $\gamma$ \\
\hline Minnesota & $0.79(0.48,1.19)$ & $1.05(0.85,1.34)$ & $\gamma$ \\
\hline Mississippi & $1.09(0.87,1.40)$ & $1.19(1.01,1.46)$ & $\gamma$ \\
\hline Missouri & $0.67(0.24,1.16)$ & $1.21(0.98,1.54)$ & $\gamma$ \\
\hline Montana & $0.92(0.75,1.18)$ & $1.01(0.71,1.41)$ & $\gamma$ \\
\hline New Hampshire & $1.01(0.75,1.34)$ & $1.35(1.09,1.73)$ & $\gamma$ \\
\hline New Jersey & $0.97(0.69,1.32)$ & $1.49(1.26,1.82)$ & $\gamma$ \\
\hline New Mexico & $1.05(0.84,1.39)$ & $1.05(0.77,1.44)$ & $\gamma$ \\
\hline New York & $1.10(0.82,1.48)$ & $1.56(1.28,1.94)$ & $\gamma$ \\
\hline Nebraska & $0.54(0.29,0.84)$ & $1.22(0.96,1.59)$ & NO HOMOG. \\
\hline Nevada & $1.03(0.71,1.50)$ & $1.27(1.10,1.51)$ & $\gamma$ \\
\hline N. Carolina & $1.17(0.91,1.55)$ & $1.36(1.14,1.66)$ & $\gamma$ \\
\hline N. Dakota & $0.66(0.41,0.97)$ & $1.25(0.98,1.67)$ & NO HOMOG. \\
\hline Ohio & $1.03(0.65,1.59)$ & $1.38(1.12,1.89)$ & $\gamma$ \\
\hline Oklahoma & $0.89(0.77,1.08)$ & $1.08(0.79,1.43)$ & $\gamma$ \\
\hline Oregon & $1.14(0.85,1.56)$ & $1.16(0.87,1.54)$ & $\gamma$ \\
\hline Pennsylvania & $1.03(0.70,1.46)$ & $1.35(1.15,1.66)$ & $\gamma$ \\
\hline Rhode Island & $1.15(0.83,1.66)$ & $1.19(0.88,1.58)$ & $\gamma$ \\
\hline S. Carolina & $1.20(0.95,1.54)$ & $1.40(1.19,1.72)$ & $\gamma$ \\
\hline
\end{tabular}


Table 7 continued

\begin{tabular}{llll}
\hline State & DPI & HCE & Homogeneity \\
\hline S. Dakota & $0.64(0.42,0.94)$ & $0.89(0.62,1.33)$ & $\boldsymbol{\gamma}$ \\
Tennessee & $1.12(0.83,1.60)$ & $1.26(1.10,1.49)$ & $\boldsymbol{\gamma}$ \\
Texas & $0.93(0.75,1.19)$ & $1.29(1.10,1.59)$ & $\boldsymbol{\gamma}$ \\
Utah & $1.28(0.88,1.99)$ & $1.33(1.04,1.76)$ & $\boldsymbol{\gamma}$ \\
Vermont & $0.87(0.58,1.26)$ & $1.36(1.11,1.74)$ & $\boldsymbol{\gamma}$ \\
Virginia & $1.26(0.94,1.73)$ & $1.33(1.14,1.64)$ & $\boldsymbol{\gamma}$ \\
W. Virginia & $1.05(0.82,1.38)$ & $1.33(1.11,1.62)$ & $\boldsymbol{\gamma}$ \\
Washington & $0.81(0.50,1.30)$ & $1.25(0.91,1.64)$ & $\boldsymbol{\gamma}$ \\
Wisconsin & $1.08(0.79,1.48)$ & $1.25(1.02,1.51)$ & $\boldsymbol{\gamma}$ \\
Wyoming & $1.15(0.97,1.47)$ & $1.06(0.91,1.32)$ & \\
\hline
\end{tabular}

Columns 2 and 3 display the estimated values of $d$ (and 95\% confidence intervals) for each series obtained from Tables 1 and 2 above. Column 4 indicates if the homogeneity condition is satisfied or not

range between 1.699 (in the case of Colorado) and 2.985 (Ohio); as for the estimates of $\mathrm{d}$, in the parametric case they are all within the $I(1)$ interval, and there are only two states with estimates significantly below 1 (Missouri, 0.63, and South Dakota, 0.62). The fact that the unit root null cannot be rejected in the majority of the states is not surprising given the wide intervals resulting from the small sample size. By contrast, the semiparametric estimates (Robinson 1995a) provide more evidence of fractional cointegration: in five states (Connecticut, Ohio, South Dakota, Vermont and Wisconsin), this hold for all three bandwidth parameters, and in a large number of states (including Delaware, Idaho, Florida, Illinois, Indiana, Kansas, Maine, Maryland, Minnesota, Missouri, New Jersey, New York, North Caroline, Oregon, South Caroline, South Dakota, Tennessee), there is at least one case of fractional cointegration.

Finally, Table 9 shows the results of the Hausman test for no cointegration of Marinucci and Robinson (2001) which compares the estimates of $d_{x}$ and $d_{y}$ (for healthcare expenditure and disposable income) with those obtained using the estimated residuals, all of them based on the semiparametric Whittle approach of Robinson (1995a). Marinucci and Robinson (2001) showed that

$$
H_{i m}=8 m\left(\hat{d}_{*}-\hat{d}_{i}\right)^{2} \rightarrow_{d} \chi_{1}^{2} \text { as } \frac{1}{m}+\frac{m}{T} \rightarrow 0,
$$

where $m<[\mathrm{T} / 2]$ is again a bandwidth parameter; $\hat{d}_{i}$ are the univariate estimates of $d_{x}$ and $d_{y}, \hat{d}_{*}$ is an estimate obtained from the residuals of the cointegrating regression. Using this approach, we find evidence of fractional cointegration in the following cases: Connecticut, Delaware, Indiana, Maine, Maryland, New Jersey, New York, Nevada, North Caroline, Tennessee and Vermont. Cointegration does not appear to hold in the remaining states. 
The relationship between healthcare expenditure and disposable...

Table 8 Estimates of $d$ for each state and homogeneity in the value of $d$

\begin{tabular}{|c|c|c|c|c|c|}
\hline \multirow[t]{2}{*}{ State } & \multirow[t]{2}{*}{$\beta_{1}(t$ value $)$} & \multirow{2}{*}{$\frac{\text { Parametric }}{d}$} & \multicolumn{3}{|c|}{ Semiparametric } \\
\hline & & & $(\mathrm{T})^{0.4}$ & $(\mathrm{~T})^{0.5}$ & $(T)^{0.6}$ \\
\hline Alabama & $2.248(57.63)$ & $0.91(0.68,1.30)$ & 0.825 & 0.751 & 0.876 \\
\hline Alaska & $2.578(6.71)$ & $1.29(1.05,1.69)$ & 0.987 & 0.997 & 1.234 \\
\hline Arizona & $2.104(28.50)$ & $1.18(0.87,1.68)$ & 0.650 & 0.742 & 0.969 \\
\hline Arkansas & $2.316(39.18)$ & $0.87(0.65,1.23)$ & 0.945 & 0.801 & 0.858 \\
\hline California & 2.193 (31.63) & $1.17(0.89,1.51)$ & 1.054 & 1.290 & 1.314 \\
\hline Colorado & 1.699 (36.32) & $1.27(0.94,1.75)$ & 1.262 & 0.942 & 1.166 \\
\hline Connecticut & 2.115 (56.58) & $0.83(0.52,1.34)$ & 0.318 & 0.500 & 0.694 \\
\hline Delaware & $2.942(43.46)$ & $0.92(0.61,1.27)$ & 0.500 & 0.394 & 0.995 \\
\hline Florida & 2.353 (41.98) & $0.92(0.66,1.38)$ & 0.260 & 0.714 & 0.691 \\
\hline Georgia & 2.109 (44.59) & $1.06(0.79,1.45)$ & 0.939 & 1.043 & 1.130 \\
\hline Hawaii & $2.807(25.04)$ & $1.08(0.86,1.37)$ & 1.500 & 1.232 & 0.981 \\
\hline Idaho & $2.734(29.55)$ & $0.80(0.59,1.10)$ & 0.777 & 1.088 & 0.712 \\
\hline Illinois & $2.431(42.91)$ & $0.83(0.54,1.19)$ & 0.300 & 1.088 & 0.712 \\
\hline Indiana & $2.845(39.22)$ & $0.65(0.37,1.07)$ & 0.237 & 0.424 & 1.015 \\
\hline Kansas & $2.378(35.05)$ & $0.90(0.68,1.20)$ & 0.606 & 0.809 & 1.049 \\
\hline Kentucky & 2.674 (41.19) & $0.92(0.72,1.21)$ & 1.219 & 1.018 & 0.949 \\
\hline Louisiana & $2.275(29.43)$ & $1.24(1.04,1.65)$ & 1.397 & 1.493 & 1.174 \\
\hline Maine & 2.530 (53.09) & $0.96(0.67,1.33)$ & 0.445 & 0.558 & 0.931 \\
\hline Maryland & $2.110(54.09)$ & $1.07(0.79,1.41)$ & 0.500 & 0.558 & 0.931 \\
\hline Massachusetts & $1.889(46.55)$ & $1.21(0.96,1.52)$ & 1.054 & 1.242 & 1.392 \\
\hline Michigan & 2.858 (26.93) & $1.11(0.73,1.62)$ & 0.946 & 0.921 & 0.834 \\
\hline Minnesota & $2.150(42.37)$ & $0.68(0.45,1.04)$ & 0.634 & 0.418 & 0.545 \\
\hline Mississippi & $2.469(51.60)$ & $0.90(0.69,1.20)$ & 1.100 & 1.165 & 0.849 \\
\hline Missouri & $2.434(48.23)$ & $0.63(0.42,0.96)$ & 0.853 & 0.418 & 0.686 \\
\hline Montana & $2.790(21.40)$ & $0.88(0.73,1.09)$ & 1.058 & 1.103 & 1.111 \\
\hline New Hampshire & 2.115 (44.46) & $0.96(0.74,1.25)$ & 0.868 & 1.205 & 1.062 \\
\hline New Jersey & $2.404(54.05)$ & $0.90(0.64,1.26)$ & 0.138 & 0.610 & 0.881 \\
\hline New Mexico & 2.609 (32.92) & $1.09(0.89,1.43)$ & 1.500 & 1.003 & 1.166 \\
\hline New York & $2.372(42.96)$ & $0.90(0.64,1.28)$ & 0.316 & 0.545 & 0.949 \\
\hline Nevada & $2.582(24.18)$ & $1.03(0.71,1.54)$ & 0.672 & 0.724 & 1.045 \\
\hline N. Carolina & $2.311(62.88)$ & $1.03(0.67,1.53)$ & -0.045 & 0.371 & 0.776 \\
\hline Ohio & 2.985 (44.27) & $0.92(0.51,1.54)$ & 0.142 & 0.280 & 0.378 \\
\hline Oklahoma & $2.345(34.78)$ & $0.92(0.77,1.14)$ & 1.488 & 1.273 & 1.257 \\
\hline Oregon & 2.457 (39.17) & $1.07(0.73,1.55)$ & 0.595 & 0.708 & 0.931 \\
\hline Pennsylvania & 2.615 (48.95) & $0.95(0.73,1.27)$ & 0.698 & 0.892 & 0.937 \\
\hline Rhode Island & $2.332(46.91)$ & $1.07(0.82,1.47)$ & 1.066 & 1.067 & 0.910 \\
\hline S. Carolina & $2.547(61.36)$ & $1.03(0.74,1.43)$ & -0.054 & 0.835 & 0.842 \\
\hline S. Dakota & $2.130(24.45)$ & $0.62(0.44,0.80)$ & 0.609 & 0.662 & 0.672 \\
\hline Tennessee & 2.118 (56.09) & $0.91(0.63,1.42)$ & 0.810 & 0.632 & 0.702 \\
\hline
\end{tabular}


Table 8 continued

\begin{tabular}{llllll}
\hline State & $\beta_{1}(t$ value $)$ & Parametric & \multicolumn{2}{l}{ Semiparametric } & \\
\cline { 3 - 5 } & & $d$ & $(\mathrm{~T})^{0.4}$ & $(\mathrm{~T})^{0.5}$ & $(T)^{0.6}$ \\
\hline Texas & $2.090(38.28)$ & $0.95(0.74,1.36)$ & 1.117 & 0.925 & 1.121 \\
Utah & $2.450(28.32)$ & $1.44(0.99,2.21)$ & 0.658 & 0.718 & 1.056 \\
Vermont & $2.268(54.46)$ & $0.84(0.56,1.23)$ & $\mathbf{0 . 5 4 2}$ & $\mathbf{0 . 5 2 9}$ & $\mathbf{0 . 6 9 5}$ \\
Virginia & $2.050(73.63)$ & $0.95(0.67,1.40)$ & $\mathbf{0 . 3 1 3}$ & 0.987 & 0.789 \\
W. Virginia & $2.894(36.53)$ & $1.00(0.79,1.30)$ & 1.260 & 0.842 & 0.970 \\
Washington & $2.162(41.95)$ & $0.94(0.68,1.35)$ & 0.641 & 1.228 & 1.101 \\
Wisconsin & $2.598(54.22)$ & $0.88(0.57,1.33)$ & $\mathbf{0 . 5 5 2}$ & $\mathbf{0 . 5 7 6}$ & $\mathbf{0 . 6 4 0}$ \\
Wyoming & $2.077(13.35)$ & $1.16(0.99,1.40)$ & 1.462 & 1.500 & 1.361 \\
\hline
\end{tabular}

The 2 nd column displays the estimated of $\beta_{1}$ (and their $t$ values) in Eq. (4) with $I(d) x_{t}$. The 3rd column displays the estimates of $d$ using the parametric approach, while the values in columns 4,5 and 6 refers to the estimates of $d$ with a semiparametric method. The confidence bands for the $I(1)$ hypothesis are $(0.632$, $1.367),(0.689,1.310)$ and $(0.739,1.269)$, respectively, for $T^{0.4}, T^{0.5}$ and $T^{0.6}$

Finally, we run OLS regressions in first differences of log healthcare expenditure on log-disposable income to shed light on the short-run income elasticities. The estimation results are displayed in Table $10 .^{2}$

In 40 states, the estimated elasticities are statistically significant and positive, ranging from 0.107 (Nebraska) to 0.752 (Georgia); in the remaining ten states (Alaska, Hawaii, Idaho, Iowa, Kansas, Oklahoma, West Virginia, Washington and Wyoming), the null of a zero slope coefficient cannot be rejected. For three states (Alabama, Georgia and South Caroline), the null of an elasticity equal to 1 cannot be rejected at the 5\% level. In brief, the evidence points to an income elasticity lower than one in most US states, which implies that health is a normal (rather than a luxury) good. Table 11 reports the estimated long-run and short-run income elasticities for healthcare expenditure in each of the 50 US states, showing in which of the states health care can be considered a luxury (Alabama, Connecticut, Delaware, Georgia, Indiana, Maine, Maryland, New Jersey, New York, Nevada, North Caroline, South Caroline, Tennessee and Vermont) or a necessity good (the rest of the states). If health care is a luxury good, demand will increase more rapidly than income, and public health policies can only have a subsidiary role. However, if it is a necessity good, more redistribution of healthcare resources and greater public involvement in health care might be needed. That is, the size of the income elasticity offers key information on the optimal level of health expenditure and on the potential role of public health policies in providing health care.

\footnotetext{
2 At this stage, it is important to point out that the short-run and the long-run elasticities displayed, respectively, in Tables 8 and 10 refer to two different models. Note that the $\beta_{1}$-coefficients presented in Table 8 refer to those based on the long-run relationship, while those in Table 10 refer to the slope and the intercept in the first differenced model. In the fractional cointegration context, we can build up a fractional VECM model as the one suggested in Johansen and Nielsen $(2010,2012)$, but under some strong assumptions, that are not incorporated in the present work. This is a possible direction in which our work could be extended in the future.
} 
The relationship between healthcare expenditure and disposable...

Table 9 Estimates of $d$ and tests of no cointegration against fractional cointegration

\begin{tabular}{|c|c|c|c|c|c|}
\hline States & $d_{x}(\mathrm{HCE})$ & $d_{y}(\mathrm{DPI})$ & $d$ (Resid.) & $H_{\mathrm{Ox}}$ & $H_{\mathrm{oy}}$ \\
\hline Alabama & 1.207 & 0.866 & 0.751 & 10.520 & 0.669 \\
\hline Alaska & 1.161 & 1.090 & 0.997 & 1.360 & 0.437 \\
\hline Arizona & 1.432 & 0.813 & 0.742 & 24.088 & 0.255 \\
\hline Arkansas & 1.361 & 0.850 & 0.801 & 15.867 & 0.121 \\
\hline California & 1.500 & 1.069 & 1.190 & 4.862 & 0.740 \\
\hline Colorado & 1.097 & 1.102 & 0.942 & 1.215 & 0.182 \\
\hline Connecticut & 1.400 & 0.898 & 0.500 & 40.983 & 8.014 \\
\hline Delaware & 1.065 & 1.286 & 0.394 & 22.780 & 40.257 \\
\hline Florida & 1.427 & 0.857 & 0.714 & 25.721 & 1.034 \\
\hline Georgia & 1.500 & 1.096 & 1.043 & 10.576 & 1.034 \\
\hline Hawaii & 1.298 & 1.500 & 1.232 & 2.231 & 0.142 \\
\hline Idaho & 1.070 & 1.500 & 1.088 & 0.016 & 8.586 \\
\hline Illinois & 1.161 & 0.887 & 0.819 & $\mathrm{xxx}$ & $\mathrm{xxx}$ \\
\hline Indiana & 1.015 & 0.649 & 0.424 & 17.672 & 5.344 \\
\hline Kansas & 0.917 & 0.823 & 0.809 & 0.590 & 0.099 \\
\hline Kentucky & 1.194 & 0.905 & 1.018 & 1.567 & 0.646 \\
\hline Louisiana & 1.415 & 1.286 & 1.193 & 2.493 & 0.437 \\
\hline Maine & 0.862 & 0.935 & 0.558 & 4.675 & 7.191 \\
\hline Maryland & 1.500 & 1.061 & 0.558 & 44.897 & 12.801 \\
\hline Massachusetts & 1.448 & 1.168 & 1.242 & 8.340 & 0.803 \\
\hline Michigan & 1.340 & 0.995 & 0.921 & 8.882 & 0.277 \\
\hline Minnesota & 1.279 & 0.680 & 0.418 & 37.508 & 3.473 \\
\hline Mississippi & 1.205 & 1.241 & 1.165 & 0.080 & 0.292 \\
\hline Missouri & 1.153 & 0.670 & 0.418 & 27.333 & 3.213 \\
\hline Montana & 1.037 & 1.111 & 1.103 & 0.220 & 0.032 \\
\hline New Hampshire & 1.314 & 1.344 & 1.205 & 0.601 & 0.977 \\
\hline New Jersey & 1.477 & 0.917 & 0.610 & 38.032 & 4.768 \\
\hline New Mexico & 0.855 & 0.864 & 0.803 & 0.136 & 0.188 \\
\hline New York & 1.450 & 0.980 & 0.545 & 41.439 & 9.574 \\
\hline Nevada & 1.411 & 1.036 & 0.724 & 54.725 & 22.375 \\
\hline N. Carolina & 1.371 & 1.048 & 0.371 & 50.596 & 23.189 \\
\hline Ohio & 1.189 & 0.500 & 0.280 & 41.806 & 2.448 \\
\hline Oklahoma & 1.331 & 1.318 & 1.273 & 0.170 & 0.102 \\
\hline Oregon & 1.183 & 0.908 & 0.708 & 11.415 & 2.023 \\
\hline Pennsylvania & 1.374 & 0.942 & 0.892 & 11.745 & 0.126 \\
\hline Rhode Island & 1.017 & 1.069 & 1.067 & 0.126 & 0.020 \\
\hline S. Carolina & 1.175 & 1.069 & 0.835 & 5.848 & 2.770 \\
\hline S. Dakota & 0.645 & 0.801 & 0.662 & $\mathrm{xxx}$ & $\mathrm{xxx}$ \\
\hline Tennessee & 1.346 & 0.933 & 0.632 & 25.793 & 4.584 \\
\hline Texas & 1.347 & 0.975 & 0.925 & 9.010 & 0.126 \\
\hline
\end{tabular}


Table 9 continued

\begin{tabular}{llllll}
\hline States & $d_{x}(\mathrm{HCE})$ & $d_{y}(\mathrm{DPI})$ & $d$ (Resid.) & $H_{\mathrm{Ox}}$ & $H_{\mathrm{Oy}}$ \\
\hline Utah & 0.954 & 0.984 & 0.718 & 2.818 & 3.580 \\
Vermont & $\mathbf{1 . 2 7 1}$ & $\mathbf{0 . 8 9 0}$ & $\mathbf{0 . 5 2 9}$ & $\mathbf{2 7 . 8 5 6}$ & $\mathbf{6 . 5 9 3}$ \\
Virginia & 1.247 & 1.267 & 0.987 & 3.420 & 3.966 \\
W. Virginia & 1.180 & 0.848 & 0.842 & 5.780 & 0.001 \\
Washington & 1.003 & 0.804 & 0.808 & 1.923 & 0.001 \\
Wisconsin & 1.500 & 0.711 & 0.576 & 43.198 & 0.922 \\
Wyoming & 1.290 & 1.306 & 1.100 & 1.826 & 2.147 \\
\hline
\end{tabular}

The values in the 2nd and 3rd columns refer to the estimated values of $d$ for the two individual series; the following column refers to the estimate of $d$ for the residuals; finally, the last two columns refers to the test statistics for $H x$ and $H y$, respectively, using the Hausman test of Marinucci and Robinson (2001). $\chi_{1}^{2}(5 \%)=3.84$. In bold are those cases where we reject the null hypothesis of no cointegration at the $5 \%$ level

Table 10 Regression based on first differences

\begin{tabular}{|c|c|c|}
\hline State & Intercept & Slope \\
\hline Alabama & $0.029(5.57)$ & $0.713(3.80)^{\mathrm{a}}$ \\
\hline Alaska & $0.042(7.63)$ & $0.018(0.14)$ \\
\hline Arizona & $0.028(5.94)$ & 0.415 \\
\hline Arkansas & $0.038(9.00)$ & $0.284(2.24)$ \\
\hline California & $0.025(5.96)$ & $0.516(2.74)$ \\
\hline Colorado & $0.029(7.35)$ & $0.122(0.79)$ \\
\hline Connecticut & $0.030(6.74)$ & 0.509 (3.39) \\
\hline Delaware & $0.036(10.83)$ & $0.413(3.08)$ \\
\hline Florida & $0.033(7.67)$ & $0.444(3.14)$ \\
\hline Georgia & $0.026(5.81)$ & $0.752(4.72)^{\mathrm{a}}$ \\
\hline Hawaii & $0.034(7.08)$ & $0.162(0.89)$ \\
\hline Idaho & $0.036(5.98)$ & 0.218 \\
\hline Illinois & $0.031(10.71)$ & $0.278(2.70)$ \\
\hline Indiana & $0.035(9.27)$ & $0.351(2.72)$ \\
\hline Iowa & $0.036(9.44)$ & $0.138(1.49)$ \\
\hline Kansas & $0.034(7.80)$ & $0.245(1.60)$ \\
\hline Kentucky & $0.037(9.77)$ & $0.358(2.72)$ \\
\hline Louisiana & $0.035(7.03)$ & $0.419(2.39)$ \\
\hline Maine & $0.038(9.04)$ & $0.428(2.78)$ \\
\hline Maryland & $0.030(6.80)$ & 0.579 (3.67) \\
\hline Massachusetts & $0.029(6.90)$ & $0.550(3.75)$ \\
\hline Michigan & $0.033(11.65)$ & $0.282(2.74)$ \\
\hline Minnesota & $0.034(8.69)$ & $0.220(1.87)$ \\
\hline Mississippi & $0.037(7.10)$ & $0.596(3.62)$ \\
\hline Missouri & $0.032(8.19)$ & $0.414(2.90)$ \\
\hline Montana & $0.035(8.11)$ & $0.308(2.31)$ \\
\hline
\end{tabular}


Table 10 continued

\begin{tabular}{lll}
\hline State & Intercept & Slope \\
\hline Nebraska & $0.036(10.56)$ & $\mathbf{0 . 2 0 6}(\mathbf{2 . 3 1})$ \\
New Hampshire & $0.036(9.18)$ & $\mathbf{0 . 3 5 6}(\mathbf{2 . 8 7})$ \\
New Jersey & $0.032(7.09)$ & $\mathbf{0 . 4 9 2}(\mathbf{2 . 9 0})$ \\
New Mexico & $0.035(7.11)$ & $\mathbf{0 . 4 4 9}(\mathbf{2 . 3 2})$ \\
New York & $0.031(8.30)$ & $\mathbf{0 . 3 6 5}(\mathbf{2 . 5 6})$ \\
Nevada & $0.028(6.21)$ & $\mathbf{0 . 5 3 4}(\mathbf{3 . 2 7})$ \\
N. Carolina & $0.031(7.64)$ & $\mathbf{0 . 6 8 7}(\mathbf{4 . 8 7})$ \\
N. Dakota & $0.038(8.00)$ & $\mathbf{0 . 1 0 7}(\mathbf{1 . 9 1})$ \\
Ohio & $0.035(9.34)$ & $\mathbf{0 . 3 4 2}(\mathbf{2 . 1 2})$ \\
Oklahoma & $0.036(8.04)$ & $0.123(0.83)$ \\
Oregon & $0.031(7.93)$ & $\mathbf{0 . 4 4 0}(\mathbf{2 . 8 1})$ \\
Pennsylvania & $0.033(8.33)$ & $\mathbf{0 . 4 6 2}(\mathbf{2 . 6 9})$ \\
Rhode Island & $0.030(7.90)$ & $\mathbf{0 . 5 7 1}(\mathbf{3 . 7 2})$ \\
S. Carolina & $0.034(7.15)$ & $\mathbf{0 . 6 6 5}(\mathbf{3 . 8 0})^{\mathrm{a}}$ \\
S. Dakota & $0.039(11.40)$ & $\mathbf{0 . 1 2 2}(\mathbf{2 . 0 5})$ \\
Tennessee & $0.029(6.73)$ & $\mathbf{0 . 6 0 0}(\mathbf{4 . 0 6})$ \\
Texas & $0.033(8.38)$ & $\mathbf{0 . 2 4 7}(\mathbf{1 . 7 4})$ \\
Utah & $0.033(7.86)$ & $\mathbf{0 . 2 5 9}(\mathbf{1 . 7 9})$ \\
Vermont & $0.033(6.87)$ & $\mathbf{0 . 3 9 1}(\mathbf{2 . 3 0})$ \\
Virginia & $0.029(6.68)$ & $\mathbf{0 . 6 4 8}(\mathbf{4 . 0 8})$ \\
W. Virginia & $0.040(8.91)$ & $0.278(1.61)$ \\
Washington & $0.032(7.78)$ & $\mathbf{0 . 5 7 0}(\mathbf{3 . 5 8})$ \\
Wisconsin & $0.031(8.36)$ & $(1.23)$ \\
Wyoming & $0.039(6.69)$ & \\
\hline & & $-0.50)$ \\
\hline & &
\end{tabular}

\section{Conclusions}

This paper examines the relationship between healthcare expenditure and disposable income in the US states over the period 1966-2009 using fractional integration and cointegration techniques. First, we estimate the fractional order of integration for each of the two series in each of the US states and find that it is equal or higher than 1 for healthcare expenditure in all states and for disposable income in most of them (except Iowa, Nebraska, North Dakota and South Dakota), which suggests that these two variables are non-stationary. These findings are confirmed when nonlinearities are introduced into the model.

Second, we test for fractional cointegration between healthcare expenditure and disposable income using various methods. The results change depending on whether a parametric or a semiparametric approach is followed. Specifically, the null of no cointegration cannot be rejected in the former case except for Missouri and South Dakota, while there is stronger evidence of cointegration in the latter case: when using the Hausman test for no cointegration of Marinucci and Robinson (2001), fractional 
G. M. Caporale et al.

Table 11 Long-run and short-run elasticities

\begin{tabular}{|c|c|c|c|}
\hline State & Long-run & Short-run & $\begin{array}{l}\text { Luxury/ } \\
\text { necessity }\end{array}$ \\
\hline Alabama & & $0.713(3.80)^{\mathrm{a}}$ & Luxury \\
\hline Alaska & & $0.018(0.14)$ & \\
\hline Arizona & & $0.415(2.61)$ & Necessity \\
\hline Arkansas & & $0.284(2.24)$ & Necessity \\
\hline California & & $0.516(2.74)$ & Necessity \\
\hline Colorado & & $0.122(0.79)$ & \\
\hline Connecticut & $2.115(56.58)$ & 0.509 (3.39) & Luxury \\
\hline Delaware & $2.942(43.46)$ & $0.413(3.08)$ & Luxury \\
\hline Florida & & $0.444(3.14)$ & Necessity \\
\hline Georgia & & $0.752(4.72)^{\mathrm{a}}$ & Luxury \\
\hline Hawaii & & $0.162(0.89)$ & Necessity \\
\hline IDAHO & & $0.218(1.08)$ & Necessity \\
\hline Illinois & & $0.278(2.70)$ & Necessity \\
\hline Indiana & $2.845(39.22)$ & $0.351(2.72)$ & Luxury \\
\hline Iowa & & $0.138(1.49)$ & \\
\hline Kansas & & $0.245(1.60)$ & \\
\hline Kentucky & & $0.358(2.72)$ & Necessity \\
\hline Louisiana & & $0.419(2.39)$ & Necessity \\
\hline Maine & $2.110(54.09)$ & $0.428(2.78)$ & Luxury \\
\hline Maryland & $1.889(46.55)$ & 0.579 (3.67) & Luxury \\
\hline Massachusetts & & $0.550(3.75)$ & Necessity \\
\hline Michigan & & $0.282(2.74)$ & Necessity \\
\hline Minnesota & & $0.220(1.87)$ & Necessity \\
\hline Mississippi & & $0.596(3.62)$ & Necessity \\
\hline Missouri & & $0.414(2.90)$ & Necessity \\
\hline Montana & & $0.308(2.31)$ & Necessity \\
\hline Nebraska & & $0.206(2.31)$ & Necessity \\
\hline New Hampshire & & $0.356(2.87)$ & Necessity \\
\hline New Jersey & $2.372(42.96)$ & $0.492(2.90)$ & Luxury \\
\hline New Mexico & & $0.449(2.32)$ & Necessity \\
\hline New York & $2.311(62.88)$ & $0.365(2.56)$ & Luxury \\
\hline Nevada & $2.985(44.27)$ & $0.534(3.27)$ & Luxury \\
\hline N. Carolina & $2.345(34.78)$ & 0.687 (4.87) & Luxury \\
\hline N. Dakota & & 0.107 (1.91) & Necessity \\
\hline Ohio & & $0.342(2.12)$ & Necessity \\
\hline Oklahoma & & $0.123(0.83)$ & Necessity \\
\hline Oregon & & $0.440(2.81)$ & Necessity \\
\hline
\end{tabular}


Table 11 continued

\begin{tabular}{llll}
\hline State & Long-run & Short-run & $\begin{array}{l}\text { Luxury/ } \\
\text { necessity }\end{array}$ \\
\hline Pennsylvania & & $\mathbf{0 . 4 6 2}(\mathbf{2 . 6 9})$ & Necessity \\
Rhode Island & $\mathbf{0 . 5 7 1 ( 3 . 7 2 )}$ & Necessity \\
S. Carolina & & $\mathbf{0 . 6 6 5}(\mathbf{3 . 8 0})^{\mathrm{a}}$ & Luxury \\
S. Dakota & & $\mathbf{0 . 1 2 2}(\mathbf{2 . 0 5})$ & Necessity \\
Tennessee & $2.268(54.46)$ & $\mathbf{0 . 6 0 0}(\mathbf{4 . 0 6})$ & Luxury \\
Texas & & $\mathbf{0 . 2 4 7}(\mathbf{1 . 7 4})$ & Necessity \\
Utah & $\mathbf{0 . 2 5 9}(\mathbf{1 . 7 9})$ & Necessity \\
Vermont & $2.162(41.95)$ & $\mathbf{0 . 3 9 1}(\mathbf{2 . 3 0})$ & Luxury \\
Virginia & & $\mathbf{0 . 6 4 8}(\mathbf{4 . 0 8})$ & Necessity \\
W. Virginia & & $0.278(1.61)$ & \\
Washington & & $0.212(1.23)$ & \\
Wisconsin & & $\mathbf{0 . 5 7 0}(\mathbf{3 . 5 8})$ & Necessity \\
Wyoming & & $-0.075(-0.50)$ & \\
\hline
\end{tabular}

Column 2 shows the long-run income elasticities for those states in which a cointegration relationship exists, and column 3 shows the short-run income elasticities, based on the regression in first differences. $t$ values in parenthesis

a We cannot reject the null of a slope coefficient equal to 1 at the $5 \%$ level. The last column shows whether health is a luxury or a necessity good in each of the US states on the basis of the estimated long-run and short-run income elasticities

cointegration is found in 11 US states (Connecticut, Delaware, Indiana, Maine, Maryland, New Jersey, New York, Nevada, North Caroline, Tennessee and Vermont).

Finally, in the US states for which cointegration holds, the income elasticity is in all cases above 1, which suggests that health care is a luxury rather than a necessity good. Elsewhere, the lack of cointegration implies that factors other than disposable income drive healthcare expenditure, and therefore, health care is instead a necessity good. As for the short-run elasticities from the regressions in first differences, in most cases they are estimated to lie in the interval $(0,1)$, being significantly positive in 40 states, while in only three states (Alabama, Georgia and South Caroline) the null hypothesis of an income elasticity equal to one cannot be rejected. The implication is that in most US states health care is a necessary good, which requires more redistribution of resources and more active health policies.

\section{References}

Acemoglu D, Finkelstein A, Notowidigdo M (2013) Income and health spending: evidence from oil price shocks. Rev Econ Stat 95:1079-1095

Ang JB (2010) The determinants of health care expenditure in Australia. Appl Econ Lett 17:639-644

Baltagi BH, Moscone F (2010) Health care expenditure and income in the OECD reconsidered: evidence from panel data. Econ Model 27:804-811

Ben Nasr A, Ajmi AN, Gupta R (2014) Modeling the volatility of the Dow Jones Islamic market world index using a fractionally integrated time varying GARCH (FITVGARCH) model. Appl Financ Econ 24:993-1004

Bierens HJ (1997) Testing the unit root with drift hypothesis against nonlinear trend stationarity with an application to the US price level and interest rate. J Econom 81:29-64

Blomqvist AG, Carter RAL (1997) Is health care really a luxury? J Health Econ 16:207-229

Cheung Y-W, Lai KS (1993) Do gold market returns have long memory? Financ Rev 28:181-202

Dahlhaus R (1989) Efficient parameter estimation for self-similar process. Ann Stat 17:1749-1766

Di Matteo L (2003) The income elasticity of health care spending. A comparison of parametric and nonparametric approaches. Eur J Health Econ 4:20-29 
Di Matteo L, Di Matteo R (1998) Evidence on the determinants of Canadian provincial government health expenditures: 1965-1991. J Health Econ 17:211-228

Diebold FX, Inoue A (2001) Long memory and regime switching. J Econom 105:131-159

Diebold FX, Rudebusch GD (1991) On the power of Dickey-Fuller test against fractional alternatives. Econ Lett 35:155-160

Dreger C, Reimers HE (2005) Health care expenditures in OECD countries: a panel unit root and cointegration analysis. IZA discussion paper series 1469

Engle RF, Granger CWJ (1987) Cointegration and error correction model. Representation, estimation and testing. Econometrica 55:251-276

Freeman DG (2003) Is health care a necessity or a luxury? Pooled estimates of income elasticity from US state-level data. Appl Econ 35:495-502

Freeman DG (2012) Is health care a necessity or a luxury? New evidence from a panel of US state-level data. Sam Houston State University Economics and International Business working paper No. 12-03

Gerdtham UG, Löthgren M (2000) On stationarity and cointegration of international health expenditure and GDP. J Health Econ 19:461-475

Gil-Alana LA (2003) Testing of fractional cointegration in macroeconomic time series. Oxf Bull Econ Stat 65:517-529

Gil-Alana LA, Hualde J (2009) Fractional integration and cointegration: An overview and an empirical application, In: Patterson K, Mills TC (eds) Palgrave handbook of econometrics, vol II. Palgrave, MacMillan, pp 434-472

Hamming RW (1973) Numerical methods for scientists and engineers, 2nd edn. McGraw-Hill, New York

Hansen P, King A (1998) Health care expenditure and GDP: panel data unit root test results, comment. J Health Econ 17:377-381

Hasslers U, Wolters J (1994) On the power of unit root tests against fractional alternatives. Econ Lett 45:1-5

Jewel T, Lee J, Tieslau M, Strazicich MC (2003) Stationarity of health expenditures and GDP: evidence from panel root tests with heterogeneous structural breaks. J Health Econ 22:313-323

Johansen S, Nielsen MØ (2010) Likelihood inference for a nonstationary fractional autoregressive model. J Econom 158:51-66

Johansen S, Nielsen MØ (2012) Likelihood inference for a fractionally cointegrated vector autoregressive model. Econometrica 80(6):2667-2732

Kleiman E (1974) The determinants of national outlay on health. Macmillan, London

Lee D, Schmidt P (1996) On the power of the KPSS test of stationarity against fractionally integrated alternatives. J Econom 73:285-302

Liu D, Li R, Wang Z (2010) Testing for structural breaks $n$ panel varying coefficient models with an application to OECD health expenditure. Empir Econ 40:95-118

MacDonald G, Hopkins S (2002) Unit root properties of OECD health care expenditure and GDP data. Health Econ 11:371-376

Marinucci D, Robinson PM (2001) Semiparametric fractional cointegration analysis. J Econom 105:225247

Narayan PK (2006) Examining structural breaks and growth rates in international health expenditures. J Health Econ 25:877-890

Newhouse J (1977) Medical care expenditure: a cross-national survey. J Hum Res 12:115-125

OECD Health Statistics (2014) http://www.oecd.org/els/health-systems/health-data.htm

Panopoulou E, Patenlidis T (2012) Convergence in per capita health expenditures and health outcomes in the OECD countries. Appl Econ 44:3909-3920

Panopoulou E, Patenlidis T (2013) Cross-state disparities in US health care expenditures. Health Econ 22:451-465

Robinson PM (1995a) Gaussian semi-parametric estimation of long range dependence. Ann Stat 23:16301661

Robinson PM (1995b) Log-periodogram regression of time series with long range dependence. Ann Stat 23:1048-1072

Robinson PM, Yajima Y (2002) Determination of cointegrating rank in fractional systems. J Econom 106:217-241

Smyth GK (1998) Polynomial approximation. Wiley, Chichester

Tomasevic NM, Stanivuk T (2009) Regression analysis and approximation by means of Chebyshev polynomial. Informatologia 42(3):166-172

Moscone F, Tosetti E (2010) Health expenditure and income in the United States. Health Econ 19:1385-1403 
Wang Z (2009) The convergence of health care expenditure in the US states. Health Econ 18:55-70

Wang Z, Rettenmaier AJ (2007) A note on cointegration of health expenditures and income. Health Econ 16:559-578

Yavuz NC, Yilanci V, Ozturk ZA (2013) Is health care a luxury or a necessity or both? Evidence from Turkey. Eur J Health Econ 14:5-10 